\title{
Recent advances in mechanical ventilation in patients with acute respiratory distress syndrome
}

\author{
Nuttapol Rittayamai ${ }^{1,2,3}$ and Laurent Brochard ${ }^{1,3}$ \\ Number 4 in the Series "Acute Respiratory Distress Syndrome” \\ Edited by S. John Wort and Stefano Nava
}

\begin{abstract}
Affiliations: ${ }^{1}$ Keenan Research Centre, St Michael's Hospital, Toronto, ON, Canada. ${ }^{2}$ Division of Respiratory Diseases and Tuberculosis, Dept of Medicine, Faculty of Medicine Siriraj Hospital, Mahidol University, Bangkok, Thailand. ${ }^{3}$ Interdepartmental Division of Critical Care Medicine, University of Toronto, Toronto, ON, Canada.
\end{abstract}

Correspondence: Laurent Brochard, St Michael's Hospital, 30 Bond Street, Toronto, ON, M5B 1W8, Canada. E-mail: brochardlasmh.ca

ABSTRACT Acute respiratory distress syndrome (ARDS) is characterised by different degrees of severity and different stages. Understanding these differences can help to better adapt the ventilatory settings to protect the lung from ventilator-induced lung injury by reducing hyperinflation or keeping the lung open when it is possible. The same therapies may be useful and beneficial in certain forms of ARDS, and risky or harmful at other stages: this includes high positive end-expiratory pressure, allowance of spontaneous breathing activity or use of noninvasive ventilation. The severity of the disease is the primary indicator to individualise treatment. Monitoring tools such as oesophageal pressure or lung volume measurements may also help to set the ventilator. At an earlier stage, an adequate lung protective strategy may also help to prevent the development of ARDS.

@ERSpublications

The survival of patients on mechanical ventilation for ARDS can be improved by optimising ventilatory management http://ow.ly/Hapm3

\section{Introduction}

Mechanical ventilation is a cornerstone in the management of patients with acute respiratory distress syndrome (ARDS). We now know that mechanical ventilation per se can aggravate lung injury, a process referred to as ventilator-induced lung injury (VILI), through several mechanisms including volutrauma, barotrauma and biotrauma [1-4]. Dynamic lung distension and repeated opening and closing of recruitable lung units are considered the two main mechanisms contributing to lung injury. The lung protective ventilation strategy using low tidal volume and limiting plateau pressure $(P$ plat $)$ has been proven to improve survival in patients with ARDS in a large randomised controlled study and was confirmed in a

Previous articles in the Series. No. 1: Guérin C. Prone ventilation in acute respiratory distress syndrome. Eur Respir Rev 2014; 23: 249-257. No. 2: Finney SJ. Extracorporeal support for patients with acute respiratory distress syndrome. Eur Respir Rev 2014; 23: 379-389. No. 3: Zompatori M, Ciccarese F, Fasano L. Overview of current lung imaging in acute respiratory distress syndrome. Eur Respir Rev 2014; 23: 519-530.

Received: Dec 152014 | Accepted after revision: Jan 052015

Conflict of interest: Disclosures can be found alongside the online version of this article at err.ersjournals.com

Provenance: Submitted article, peer reviewed.

Copyright OERS 2015. ERR articles are open access and distributed under the terms of the Creative Commons Attribution Non-Commercial Licence 4.0. 
meta-analysis $[5,6]$. Current research still suggests that further reducing ventilator-associated or -induced lung injury is the main avenue to further reduce mortality in this syndrome.

For a clinician in 2015, the main consequences of making the diagnosis of ARDS concern the supportive therapy he or she will apply to the patient. The clinician is essentially managing the consequences of ARDS. These result from three major physiological derangements: 1) a major defect in oxygenation; 2) a poor efficiency of the lungs at eliminating $\mathrm{CO}_{2}$; and 3) a major reduction in lung volumes and compliance making ARDS a severe restrictive lung disease. For each component the key issue in daily management is the price to pay to improve the physiological consequences of these problems [5]. It took many years to realise that the price of normalising physiological parameters was often unacceptably high and to put the problem of VILI central to our clinical approach [7]. Thus, evaluating the three components is essential since the management strategy may be based on this assessment: 1) assessment of gas exchange and oxygenation, which includes the influence of haemodynamics or intracardiac shunt; 2) dead space, which can be assessed grossly by calculating the "corrected" level of minute ventilation that would be needed for normalising arterial $\mathrm{CO}_{2}$ tension [8]; and 3) lung volume, assessed indirectly through the decrease in compliance or directly by measuring end-expiratory lung volume [9]. Measurement of oesophageal pressure ( $P_{\text {oes }}$ ) as a surrogate for pleural pressure helps to determine the lung mechanics and separate the effect of the chest wall [10]. In addition, the assessment of lung recruitability may be of great help to individualise the settings for mechanical ventilation and choose the level of positive end-expiratory pressure (PEEP) needed to keep the lung sufficiently open to minimise the risks of repeated opening and closing of alveoli. In addition, we now have better knowledge of what may worsen ARDS and this knowledge has been used to try to prevent the development of ARDS.

An individualisation of therapy is essential since application of the same concepts may be beneficial in certain forms of ARDS or at certain stages, and may be risky or harmful in others. This is the case for spontaneous breathing activity, noninvasive ventilation (NIV) and high PEEP, which we will discuss below.

\section{Preventing ARDS}

Early identification of patients at risk for developing ARDS and implementation of preventive strategies becomes an important approach for critically ill patients admitted to intensive care units (ICU), particularly patients receiving mechanical ventilation. ARDS is not frequently present at the time of visiting the emergency room or hospital admission and it frequently occurs over a period of hours to days following the clinical insult [11-14]. Many clinical risk factors are associated with the development of ARDS such as sepsis, shock, pneumonia, pancreatitis, aspiration, high-risk trauma and surgery, and multiple blood transfusions. A large multicentre observation study demonstrated that ARDS develops within a median of 2 days after hospital admission and also markedly increases in-hospital mortality in this patient population [14]. Many patients, therefore, develop ARDS during hospital admission because of a second-hit, but from potentially preventable exposures. AHMED et al. [11] demonstrated that inadequate antimicrobial therapy, medical and surgical adverse events, hospital-acquired aspiration, ventilation with high tidal volumes, and greater volumes of blood transfusion and fluid administration were significantly associated with the secondary development of ARDS. Prompt resuscitation to reach the goal within $6 \mathrm{~h}$, early administration of effective antimicrobials, and appropriate source control could help to prevent the development of ARDS. Similarly, optimal fluid and transfusion management (i.e. early fluid resuscitation during periods of septic shock and avoiding positive fluid balance after periods of shock) and monitoring of fluid balance, such as extravascular lung water, in mechanically ventilated patients might prevent the development of ARDS.

A lung protective ventilation strategy using low tidal volume and limiting $P_{\text {plat }}$ is now proposed in patients with ARDS. Potentially injurious ventilator settings might also be associated with secondary development of ARDS in mechanically ventilated patient without ARDS at the onset. Observational studies demonstrated that high tidal volume and Pplat are major risk factors for the secondary development of ARDS $[15,16]$. Thus, the lung protective ventilation strategy may also prevent lung injury in mechanically ventilated patients without ARDS. A randomised controlled study comparing low versus conventional tidal volumes (6 versus $10 \mathrm{~mL} \cdot \mathrm{kg}^{-1}$ predicted body weight (PBW)) in critically ill patients without acute lung injury at the onset of mechanical ventilation found that the level of systemic inflammatory cytokines decreased significantly in the low tidal volume group and development of lung injury was higher in the conventional tidal volume group (13.5 versus $2.6 \% ; \mathrm{p}=0.01)$ [17]. A recent systematic review and meta-analyses suggested that using lower tidal volumes ( $\leqslant 6$ versus $\left.\geqslant 10 \mathrm{~mL} \cdot \mathrm{kg}^{-1} \mathrm{PBW}\right)$ in patients without ARDS was associated with better clinical outcomes, including development of ARDS, mortality and duration of mechanical ventilation [16]. Strong evidence is still lacking, but it is possible that implementing the lung protective strategy and also applying an appropriate PEEP level may be needed in patients requiring mechanical ventilation in whom it is expected to be required for $>48 \mathrm{~h}$. 


\section{NIV in ARDS}

NIV has been increasingly used in the ICU as a first-line therapy for $15-20 \%$ of critically ill patients with acute respiratory failure $[18,19]$. NIV has demonstrated survival benefits in specific patient groups including acute exacerbations of chronic obstructive pulmonary disease and acute cardiogenic pulmonary oedema, and for facilitating extubation in hypercapnic respiratory failure. In acute hypoxaemic respiratory failure, a meta-analysis from five randomised studies found that NIV significantly reduced the rate of intubation and also mortality rate [20]. The heterogeneity of diagnoses of hypoxaemic respiratory failure, however, makes it difficult to interpret the benefits of NIV in this patient group, and a specific risk of delaying intubation has also been described in this group [21]. ARDS is one of the most severe forms of hypoxaemic respiratory failure and the role of NIV is still inconclusive. A multicentre, randomised study by DeLCLAux et al. [22] comparing continuous positive airway pressure (CPAP) with standard oxygen therapy in 123 patients $(83 \%$ were patients with acute lung injury) failed to demonstrate any benefit of CPAP in terms of intubation rate, ICU length of stay, and ICU and hospital mortality. In addition, patients receiving NIV developed a higher number of adverse events than standard oxygen therapy. A multicentre cohort study by ANTONELLI et al. [23] surveying the use of NIV as first-line therapy for ARDS demonstrated that NIV was followed by intubation in $46 \%$ of patients. A high SAPS (Simplified Acute Physiology Score) II score and an arterial $\mathrm{O}_{2}$ tension $\left(\mathrm{PaO}_{2}\right)$ to inspiratory $\mathrm{O}_{2}$ fraction $\left(\mathrm{FiO}_{2}\right)$ ratio $\leqslant 175$ after $1 \mathrm{~h}$ of NIV were associated with NIV failure and the requirement for endotracheal intubation. A recent observational study by THILLE et al. [24] in 113 patients requiring NIV for hypoxaemic respiratory failure (82 patients with ARDS) found that ARDS patients had a significantly higher intubation and ICU mortality rate than non-ARDS patients. $\mathrm{PaO}_{2} / \mathrm{FiO}_{2}<150$ was associated with a high intubation rate. Thus, the evidence for using NIV in patients with ARDS is limited, and may be discussed probably only in mild-to-moderate ARDS patients $\left(\mathrm{PaO}_{2} / \mathrm{FiO}_{2}>150\right)$. The risk of VILI associated with the large tidal volumes favoured by NIV probably exists, although this has not been proven [21]. In addition, close monitoring and prompt intubation in patients without improvement in oxygenation and clinical parameters within the first hour should be considered.

\section{Spontaneous versus passive breathing in ARDS}

Passive mechanical ventilation in ARDS

The lung protective ventilation strategy is now proposed as standard care for patients presenting with ARDS by using low tidal volume of $6 \mathrm{~mL} \cdot \mathrm{kg}^{-1} \mathrm{PBW}$ and limiting the inspiratory $P$ plat to $<28-30 \mathrm{cmH}_{2} \mathrm{O}$. Implementation of the lung protective ventilation strategy in patients exhibiting high respiratory drive and work of breathing may often lead to development of patient-ventilator dyssynchrony, such as double triggering, despite administration of sedative and analgesic agents [25]. Neuromuscular blocking agents (NMBAs) have been used for decades to correct this problem and can facilitate low tidal volume ventilation. Data from several randomised controlled studies in ARDS demonstrated that 25-55\% of patients routinely received a NMBA [26-28]. Concerns about the safety of administration have been raised, especially since we know that diaphragm atrophy can rapidly occur in case of disuse. GAINNIER et al. [29] conducted a pilot randomised controlled study by enrolling 56 patients with moderate-to-severe ARDS $\left(\mathrm{PaO}_{2} / \mathrm{FiO}_{2}<150\right)$ to receive cisatracurium or placebo for $48 \mathrm{~h}$. They showed that patients in the NMBA group had a sustained improvement in oxygenation and a lower $P$ plat after $48 \mathrm{~h}$ of randomisation. A second randomised controlled study by Forel et al. [30] demonstrated the same result associated with a decreased concentration of proinflammatory cytokines (interleukin (IL)-1 $\beta$, IL-6 and IL-8) in both bronchoalveolar lavage fluid and serum. A larger multicentre randomised controlled trial (the ACURASYS study) by the same group enrolled 340 patients with moderate-to-severe ARDS $\left(\mathrm{PaO}_{2} / \mathrm{FiO}_{2}<150\right.$ with PEEP $>5 \mathrm{cmH}_{2} \mathrm{O}$ ) presenting within $48 \mathrm{~h}$ to receive cisatracurium or placebo for $48 \mathrm{~h}$ [31]. The early administration of cisatracurium reduced adjusted 90-day mortality and barotrauma, and also increased ventilator free days without increasing muscle weakness (although this was assessed subjectively). In a post hoc analysis, the mortality benefit of cisatracurium was limited to patients with a $\mathrm{PaO}_{2} / \mathrm{FiO}_{2}$ ratio $<120$. The important concern about using NMBAs is the development of ICU-acquired weakness, which led to long-term morbidity in patient with ARDS $[32,33]$. The data from both experimental and clinical studies suggest that cisatracurium is not associated with ICU-acquired weakness when used for a short period of time. The mechanisms of NMBAs for improving gas exchange and outcomes are not completely clear [34]. Proposed mechanisms include minimising VILI by minimising the transpulmonary pressure changes during assisted breathing and reducing patient-ventilator asynchrony, decreasing oxygen consumption of respiratory muscles, and reducing pulmonary and systemic inflammation. Thus, NMBAs should be considered for early and short-term use in patients with severe ARDS.

\section{Maintaining spontaneous breathing in ARDS}

Complete inactivity of the diaphragm results in disuse atrophy and muscle weakness, referred to as ventilator-induced diaphragmatic dysfunction (VIDD), after as little as 18-24 h of mechanical ventilation [35, 36]. 
This can contribute to weaning problems and poorer prognosis. In experimental studies, allowance of spontaneous breathing using either assist-control ventilation or pressure support ventilation can reduce VIDD [37, 38]. The place and timing of spontaneous breathing in patients with ARDS has been debated for several years. Experimental lung injury models of ARDS demonstrated that preserving spontaneous breathing was associated with: 1) reduced markers of lung inflammation and epithelial cell damage; 2) improved tidal ventilation, gas exchange and oxygen delivery; and 3) increased systemic blood flow. Neumann et al. [39] and Putensen et al. [40] demonstrated that partial ventilatory support with airway pressure release ventilation (APRV) promoted alveolar recruitment in juxta-diaphragmatic areas, improved ventilation/perfusion matching and gas exchange, and increased oxygen delivery in comparison with controlled mechanical ventilation. It should be noted that among the studies described above, the study populations had a mild-to-moderate degree of lung injury. The impact of spontaneous breathing in severe ARDS may be completely different and may be harmful. YosHIDA and co-workers [41, 42] found that spontaneous breathing in a model of severe lung injury caused high transpulmonary pressure, worsened oxygenation and lung damage, and could also cause local injury by internal redistribution of volume. Thus, from the body of evidence, allowance of spontaneous breathing should be limited to mild-to-moderate ARDS. By contrast, early use of NMBAs should be considered in patients with severe ARDS.

One important consideration when a patient with ARDS generates spontaneous breathing efforts is that the real transpulmonary pressure becomes the sum of the pressure generated by the ventilator and by the patient's respiratory muscles. Clinicians should be aware that, when using pressure-targeted modes of ventilation including pressure assist/control ventilation (PACV) and pressure support ventilation, the true driving pressure is higher than the airway pressure $\left(P_{a w}\right)$ displayed by the ventilator. This scenario is different from volume-controlled ventilation in which transpulmonary pressure and tidal volume are kept constant irrespective of muscular pressure (fig. 1). In addition, during PACV, when the patient makes a strong effort, transpulmonary pressure and tidal volume increase substantially which may be potentially harmful (fig. 2). In an experimental and clinical study by RichARD et al. [43], nonsynchronised pressure-targeted modes of ventilation, such as APRV, resulted in lower tidal volumes and transpulmonary pressure than fully synchronised and partially synchronised pressure-targeted modes of ventilation, such as PACV and biphasic airway pressure, despite similar pressure settings on the ventilator and the patient's effort. An ongoing large multicentre randomised controlled study (Early Spontaneous Breathing in Acute Respiratory Distress Syndrome (BiRDS) study; ClinicalTrials.gov identifier: NCT01862016) will examine the efficacy and safety of early spontaneous breathing with APRV mode using normal inspiratory to expiratory ratios in comparison with controlled mechanical ventilation. The evidence for new modes of ventilation including proportional assist ventilation and neurally adjusted ventilatory assist, in which the patient selects his/her own tidal volume, and noisy pressure support ventilation, which introduces variability, is limited to experimental or physiological studies and still lack clinical data.

In summary, in severe ARDS complete relaxation of the respiratory muscles may be needed to ensure lung protection, while in less severe forms or at a later stage spontaneous breathing should be allowed with great caution to minimise the risk of amplifying transpulmonary pressure. A nonsynchronised mode may be preferable in this regard.
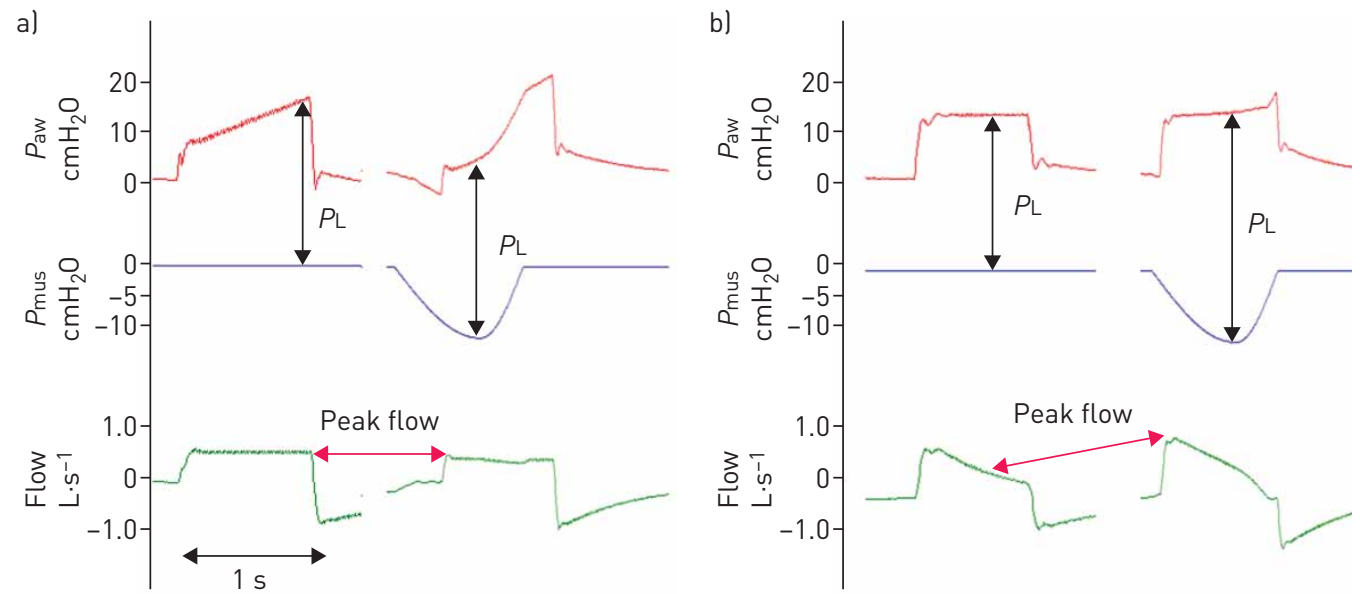

FIGURE 1 Change in transpulmonary pressure $\left(P_{\mathrm{L}}\right)$ from passive to active breathing during a) volume control ventilation (VCV) and b) pressure control ventilation (PCV). By contrast to VCV, with PCV an increasing effort will increase the flow and volume delivered. $P$ aw: airway pressure; $P$ mus: muscular pressure. 


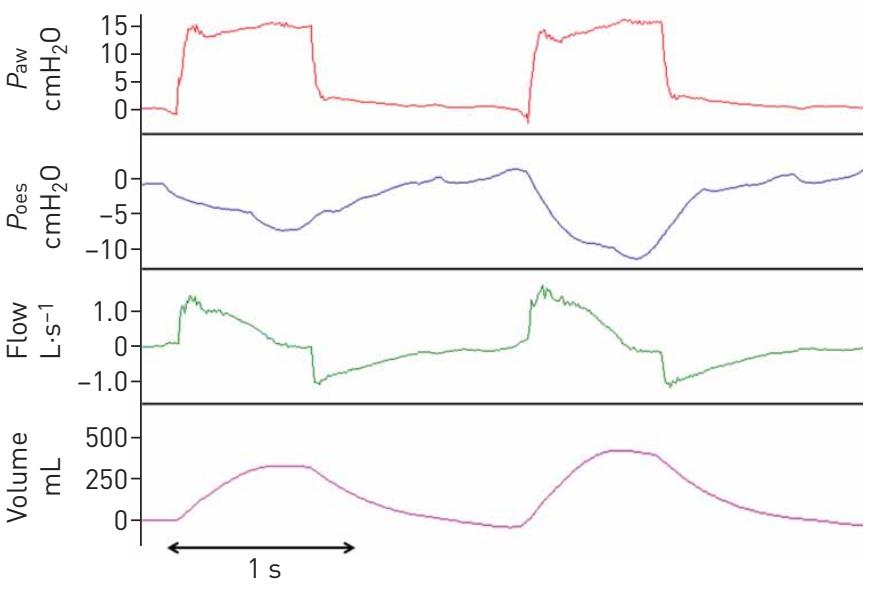

FIGURE 2 Effect of increasing inspiratory effort on delivered tidal volume during pressure-targeted ventilation. Paw: airway pressure; Poes: oesophageal pressure.

\section{Titrating PEEP}

ARDS is characterised by a major loss of lung volume due to alveolar flooding, atelectasis and consolidation. Since the first description of ARDS, the use of PEEP has been proposed to combat hypoxaemia and atelectasis [44]. Three large multicentre randomised trials have tested higher versus lower levels of PEEP while limiting tidal volumes in all patients: ALVEOLI (Assessment of Low tidal Volume and elevated End-expiratory volume to Obviate Lung Injury) [45], ExPress (Expiratory Pressure) [28], and LOVS (Lung Open Ventilation Study) [27]. They found no significant improvement in mortality, although the ExPress trial found an improvement in ventilator-free days [28]. An individual patient-based meta-analysis [26] combining these data, however, found a modest but significant reduction in mortality for patients with moderate and severe ARDS assigned to higher PEEP. There is ongoing research into how best to individualise PEEP at the bedside and to set high PEEP only in those who will benefit.

The aim of PEEP in ARDS is to recruit (or maintain recruitment of) atelectatic or flooded lung. Initial studies looking at the effects of PEEP described adverse haemodynamic effects at high levels, leading to a reduction in cardiac output and oxygen delivery [46-48]. In the best cases, PEEP is able to keep the recruited lung areas reopened by the ventilator, and thus improve gas exchange in patients with ARDS and reduce the risk of repeated opening and closure [44]. A wide variability in the amount of recruitable lung exists among patients, and between $0 \%$ and $50 \%$ of potentially recruitable lung has been described [49]. High PEEP may be able to keep the lung open only if the lung is recruitable. A major challenge to protocolising PEEP titration is, therefore, the heterogeneity with which patients respond. While effective application of higher PEEP will reduce alveolar stress and improve gas exchange, it can fail to recruit or be harmful causing regional lung over-distension or haemodynamic depression. Therefore, a positive response to increased PEEP is expected in some, but not all, patients. The impact of PEEP on VILI and mortality may depend on this response.

The main physiological consequence of PEEP is the increase in end-expiratory lung volume (EELV). In a highly recruitable patient, a substantial part of the increase in EELV can be due to reopening of previously collapsed lung tissue, referred to as recruitment. This will have potential benefits and minimal risk. In a poorly recruitable patient, most of the increase in EELV is generated by inflation of previously open lung tissue potentially leading to overdistension (volutrauma, a major mechanism of VILI [50,51]), and with very little potential benefit, failing to recruit the collapsed tissue. Therefore, the potential effectiveness and benefit of high PEEP levels depends on the patient's recruitability.

Clinical trials have suggested that a similar target for PEEP settings in all ARDS patients without a valid assessment of individual recruitability may not be very efficient for improving survival [27, 28, 45]. This may explain why the effect in terms of improved survival with high PEEP originating from randomised controlled trials is small [26]. In this regard, assessment of the individual recruitability may be essential for individualised PEEP settings to better decide the PEEP level based on the beneficial and deleterious effects of PEEP. Different techniques have been proposed: multiple pressure-volume curves, measurement of lung volume, use of Poes and transpulmonary pressure, use of lung ultrasound and use of a physiological test based on oxygenation. In research studies, alveolar recruitability has been assessed using computed tomography (CT) [52]. This technique necessitates scanning patients at different static pressure levels (45 $\mathrm{cmH}_{2} \mathrm{O}$ and 5-15 $\mathrm{cmH}_{2} \mathrm{O}$, respectively) and a detailed analysis of the CT scan cuts to compare aeration [49]. Although it provides the most visual way to detect recruitability, the individual analysis of 
each CT scan cut is extremely time-consuming and not applicable to clinical practice. It also exposes patients to the risk of repeated radiation exposure.

\section{Multiple pressure-volume curves technique}

This approach consists of plotting several pressure-volume curves obtained at different PEEP levels on the same volume axis, measuring or estimating the volume above functional residual capacity (FRC), i.e. the relaxation volume at zero end-expiratory pressure (ZEEP), at each PEEP level [53, 54]. Elastic pressurevolume curves can be obtained using low flow inflation. With this technique it is then possible to compare the lung volume above FRC in different conditions but always at the same pressure (e.g. 15 or $20 \mathrm{cmH}_{2} \mathrm{O}$ ). The change in lung volume induced by PEEP can be measured by a prolonged expiration from higher PEEP to lower PEEP or FRC. When reducing PEEP to ZEEP during a prolonged expiration, the lung volume expired is the volume above FRC at that PEEP level. Numerous studies have demonstrated good reproducibility of pressure-volume curves for assessment of alveolar recruitability [55-57]. Due to its relative complexity, however, this technique often remains limited to research areas.

\section{Poes monitoring}

Measuring Poes to estimate pleural pressure and then estimating transpulmonary pressure at end-inspiration and expiration from the difference between $P$ plat or PEEP and oesophageal pressures is a proposed method to titrate PEEP and adjust pressures: transpulmonary pressure $=P$ aw $-P$ oes [10]. The usefulness of $P_{\text {oes }}$ in guiding PEEP therapy in ARDS has been shown in the EPVent (Esophageal Pressure directed Ventilation) study [58]. Because of reduced chest wall compliance, oedema or abdominal distension, Poes is often elevated in patients with ARDS and the calculated transpulmonary pressure can be negative at end-expiration. This may indicate closed or compressed airways or atelectatic lung. Thus, PEEP could be increased until transpulmonary pressure becomes positive at end-expiration to keep the airways open (with the caveat that positive values do not assure open alveoli in the zones distal to the sampling catheter). In a single centre, randomised controlled trial, investigators compared mechanical ventilation guided by $P_{\text {oes }}$ measurements (experimental arm) with ventilation based on the protocol of the US National Institutes of Health sponsored ARDSNetwork (control arm) [5]. Patients who had PEEP titrated to ensure a positive end-expiratory transpulmonary pressure experienced a higher $\mathrm{PaO}_{2} / \mathrm{FiO}_{2}$, better respiratory system compliance as a possible consequence of improved recruitment, and a trend towards reduced 28-day mortality. This research may be considered proof of concept for the usefulness of Poes measurements in ARDS but more data are needed before widespread clinical use.

Other investigators have used an elastance-based method to estimate transpulmonary pressure, which neglects the absolute values and relies on the tidal Poes swings to calculate chest wall elastance [59]. This method estimates the lung distending pressure applied by positive pressure inflation during mechanical ventilation, i.e. eliminating the influence of the chest wall. Since any positive pressure applied at the airway opening acts on two elastic structures connected in series (the lung and the chest wall), $P$ aw is distributed between chest wall and lung elastance. The ratio of lung to respiratory system elastance can be used to better interpret the effect of Paw. The latter method for partitioning lung and chest wall elastance has been used to guide a transpulmonary "open lung" approach in a cohort of patients with severe ARDS related to influenza A (H1N1) [60]. This assessment helped clinicians to decide, in severely hypoxaemic patients requiring high $P$ aw pressures, whether it was appropriate to further increase pressures on the ventilator or whether an extracorporeal oxygenation technique was preferable. While further studies are needed to test alternative methods of calculating transpulmonary pressure, the results from such studies support the use of $P$ oes measurement in sedated and paralysed subjects when titrating ventilator settings in ARDS.

\section{Lung volume measurement using the nitrogen washout/wash-in technique}

Direct measurement of lung volume is now feasible and allows measurement of FRC and/or EELV at each PEEP level and calculation of the strain, i.e. the change in lung volume relative to FRC. Recently, washout/ wash-in techniques using nitrogen or $\mathrm{O}_{2}$ and $\mathrm{CO}_{2}$ sensors have been available in ICU ventilators, allowing bedside lung volume measurement $[9,61]$. The washout/wash-in technique has shown good correlations with helium dilution or CT scans for EELV measurement [62]. The technique uses a change in $\mathrm{FiO}_{2}$ to allow the calculation of nitrogen washout and then wash-in of the aerated lung volume. Although measuring changes in lung volume by itself is not sufficient to assess recruitment, a relatively simple method has been described as follows. Comparison of the change in lung volume with the expected change in lung volume based on the change in pressure and the static compliance gives a reasonable estimate at the bedside of the amount of recruitment occurring when changing PEEP [9]. Thus, this technique can directly quantify alveolar recruitment. 


\section{Lung ultrasound}

Some interesting data suggest that evaluating the response to PEEP could be done by assessing the lung reaeration with lung ultrasound. BOUHEMAD et al. [63] have used a specific score based on the repeated examination of six lung regions in each lung, before and after increasing PEEP. Their results suggested that the transthoracic lung ultrasound technique is a method equivalent to the pressure-volume curve method for quantitative assessment of PEEP-induced lung recruitment. More data will be needed to assess the reproducibility and feasibility of this technique, but its noninvasive nature is attractive.

\section{Oxygenation response to PEEP}

Clinicians use oxygenation to titrate PEEP, but we know this has many drawbacks including the possible presence of intracardiac shunt, the influence of haemodynamics, and a significant but relatively poor correlation existing between recruitment and oxygenation [48, 55, 64]. The oxygenation response after a change in PEEP varies widely. The preceding randomised controlled trials assessing PEEP often tried to increase oxygenation based on a fixed target and a progressive increase in PEEP. They did not try to offer high PEEP to responders and low PEEP to nonresponders. A recent post hoc analysis of the published trials suggested that among patients in whom PEEP was increased after randomisation, the higher the increase in oxygenation after PEEP the higher the reduction in mortality associated with PEEP. These findings suggest that a better way to design a trial with PEEP may be to use the physiological response to PEEP as a marker of the need for high PEEP.

In summary, high PEEP has been shown to be beneficial in patients with ARDS. A better individualisation of therapy using bedside methods to assess recruitability seems necessary. Changes in lung volume, multiple pressure-volume curves and lung ultrasound are attractive methods. The oxygenation response to PEEP may also be used as a guide for titration. These approaches will need further validation.

\section{Reducing the burden of ventilation by extracorporeal $\mathrm{CO}_{2}$ removal}

Extracorporeal $\mathrm{CO}_{2}$ removal $\left(\mathrm{ECCO}_{2} \mathrm{R}\right)$ uses a veno-venous (or arterio-venous) extracorporeal device at low blood flow rates $\left(300-1000 \mathrm{~mL} \cdot \mathrm{min}^{-1}\right)$. This low flow rate is adequate for substantial $\mathrm{CO}_{2}$ removal but will allow only minimal blood oxygenation $[65,66]$. Types of cannulae and insertion location vary, and are currently evolving. If proven to be effective, $\mathrm{ECCO}_{2} \mathrm{R}$ could potentially be used in an approach that is similar to continuous renal replacement techniques and be available in most ICUs. $\mathrm{ECCO}_{2} \mathrm{R}$ could be used technically like a simple continuous veno-venous haemofiltration circuit, but for which the primary goal would be to eliminate $\mathrm{CO}_{2}$ from the blood. The major difference with the technique described as venovenous extracorporeal membrane oxygenation (ECMO) is that much lower blood flows are needed to remove $\mathrm{CO}_{2}$, compared with 3-5 L.min ${ }^{-1}$ with ECMO. The advantage of the low flow is that relatively small vascular cannulas can be used for this amount of blood flow. Potentially, removing $\mathrm{CO}_{2}$ with an extracorporeal device could facilitate generalised use of protective or ultraprotective lung ventilation in many patients with ARDS. In addition, the decrease in tidal volume, down to $4 \mathrm{~mL} \cdot \mathrm{kg}^{-1} \mathrm{PBW}$, could facilitate an increase in PEEP.

In a prospective cohort study, TERRAGNi et al. [66] used a $\mathrm{CO}_{2}$ removal device to reduce tidal volume to $<6 \mathrm{~mL} \cdot \mathrm{kg}^{-1}$ PBW and observed an improvement of morphological markers of lung protection. A randomised control trial compared a ventilation strategy with a very low tidal volume $\left(3 \mathrm{~mL} \cdot \mathrm{kg}^{-1} \mathrm{PBW}\right)$ combined with a pump-less $\mathrm{ECCO}_{2} \mathrm{R}$ device versus conventional protective ventilation in 79 patients with severe ARDS [65]. Ventilation with $3 \mathrm{~mL} \cdot \mathrm{kg}^{-1} \mathrm{PBW}$ combined with arterio-venous $\mathrm{ECCO}_{2} \mathrm{R}$ was safe and feasible, and did not result in physiologically relevant hypercapnia/acidosis. The use of extracorporeal $\mathrm{CO}_{2}$ elimination was associated with a significant reduction in analgesic and sedative use, and resulted in an increased ratio of spontaneous breathing compared with controls. The serum levels of the pro-inflammatory cytokine IL-6 were significantly reduced early in the study period in patients treated with very low tidal volumes compared with control patients. Mechanical ventilation using lower tidal volumes and extracorporeal $\mathrm{CO}_{2}$ removal was not associated with a significant reduction of mechanical ventilation or ICU and hospital stay, but a post hoc analysis indicated that ARDS patients who were more hypoxaemic $\left(\mathrm{PaO}_{2} / \mathrm{FiO}_{2}<150\right)$ and treated with the low tidal volume strategy had a significantly shorter ventilation period [65]. Some complications were noted. This approach is, therefore, promising but it is too early for it to be applied.

\section{Conclusion}

Major advances have been made in our understanding of the pathophysiological abnormalities present at the different stages of ARDS. We have evidence for the benefits of using high PEEP but also understand that it needs to be based on a more individualised response. Spontaneous breathing may need to be avoided at an early and severe stage, while it may be favoured cautiously in milder and later stages of ARDS. New monitoring tools, including Poes measurements may considerably help in this regard. 


\section{References}

1 De Prost N, Dreyfuss D. How to prevent ventilator-induced lung injury? Minerva Anestesiol 2012; 78: 1054-1066. Ricard JD, Dreyfuss D, Saumon G. Ventilator-induced lung injury. Eur Respir J 2003; 22: Suppl. 42, 2s-9s.

Dreyfuss D, Saumon G. From ventilator-induced lung injury to multiple organ dysfunction? Intensive Care Med 1998; 24: 102-104.

4 Dreyfuss D, Saumon G. Ventilator-induced lung injury: lessons from experimental studies. Am J Respir Crit Care Med 1998; 157: 294-323.

5 Ventilation with lower tidal volumes as compared with traditional tidal volumes for acute lung injury and the acute respiratory distress syndrome. The Acute Respiratory Distress Syndrome Network. N Engl J Med 2000; 342: 1301-1308.

6 Burns KE, Adhikari NK, Slutsky AS, et al. Pressure and volume limited ventilation for the ventilatory management of patients with acute lung injury: a systematic review and meta-analysis. PLoS One 2011; 6: e14623.

7 Dreyfuss D, Saumon G. Ventilation-induced injury. In: Tobin MJ, ed. Principles and Practice of Mechanical Ventilation. New York, McGraw Hill, 1994; pp. 793-811.

8 Wexler HR, Lok P. A simple formula for adjusting arterial carbon dioxide tension. Can Anaesth Soc J 1981; 28: $370-372$.

9 Dellamonica J, Lerolle N, Sargentini C, et al. PEEP-induced changes in lung volume in acute respiratory distress syndrome. Two methods to estimate alveolar recruitment. Intensive Care Med 2011; 37: 1595-1604.

10 Akoumianaki E, Maggiore SM, Valenza F, et al. The application of esophageal pressure measurement in patients with respiratory failure. Am J Respir Crit Care Med 2014; 189: 520-531.

11 Ahmed AH, Litell JM, Malinchoc M, et al. The role of potentially preventable hospital exposures in the development of acute respiratory distress syndrome: a population-based study. Crit Care Med 2014; 42: 31-39.

12 Gajic O, Dara SI, Mendez JL, et al. Ventilator-associated lung injury in patients without acute lung injury at the onset of mechanical ventilation. Crit Care Med 2004; 32: 1817-1824.

13 Gajic O, Frutos-Vivar F, Esteban A, et al. Ventilator settings as a risk factor for acute respiratory distress syndrome in mechanically ventilated patients. Intensive Care Med 2005; 31: 922-926.

14 Shari G, Kojicic M, Li G, et al. Timing of the onset of acute respiratory distress syndrome: a population-based study. Respir Care 2011; 56: 576-582.

15 Serpa Neto A, Simonis FD, Barbas CS, et al. Association between tidal volume size, duration of ventilation, and sedation needs in patients without acute respiratory distress syndrome: an individual patient data meta-analysis. Intensive Care Med 2014; 40: 950-957.

16 Sinha P, Sanders RD, Soni N, et al. Acute respiratory distress syndrome: the prognostic value of ventilatory ratio a simple bedside tool to monitor ventilatory efficiency. Am J Respir Crit Care Med 2013; 187: 1150-1153.

17 Determann RM, Royakkers A, Wolthuis EK, et al. Ventilation with lower tidal volumes as compared with conventional tidal volumes for patients without acute lung injury: a preventive randomized controlled trial. Crit Care 2010; 14: R1.

18 Demoule A, Girou E, Richard JC, et al. Increased use of noninvasive ventilation in French intensive care units. Intensive Care Med 2006; 32: 1747-1755.

19 Esteban A, Frutos-Vivar F, Muriel A, et al. Evolution of mortality over time in patients receiving mechanical ventilation. Am J Respir Crit Care Med 2013; 188: 220-230.

20 Hess DR. The evidence for noninvasive positive-pressure ventilation in the care of patients in acute respiratory failure: a systematic review of the literature. Respir Care 2004; 49: 810-829.

21 Brochard L, Lefebvre JC, Cordioli RL, et al. Noninvasive ventilation for patients with hypoxemic acute respiratory failure. Semin Respir Crit Care Med 2014; 35: 492-500.

22 Delclaux C, L'Her E, Alberti C, et al. Treatment of acute hypoxemic nonhypercapnic respiratory insufficiency with continuous positive airway pressure delivered by a face mask: a randomized controlled trial. JAMA 2000;284 2352-2360

23 Antonelli M, Conti G, Moro M, et al. Predictors of failure of noninvasive positive pressure ventilation in patients with acute hypoxemic respiratory failure: a multi-center study. Intensive Care Med 2001; 27: 1718-1728.

24 Thille AW, Contou D, Fragnoli C, et al. Non-invasive ventilation for acute hypoxemic respiratory failure: intubation rate and risk factors. Crit Care 2013; 17: R269.

25 Kallet RH, Luce JM. Detection of patient-ventilator asynchrony during low tidal volume ventilation, using ventilator waveform graphics. Respir Care 2002; 47: 183-185.

26 Briel M, Meade M, Mercat A, et al. Higher vs lower positive end-expiratory pressure in patients with acute lung injury and acute respiratory distress syndrome: systematic review and meta-analysis. JAMA 2010; 303: 865-873.

27 Meade MO, Cook DJ, Guyatt GH, et al. Ventilation strategy using low tidal volumes, recruitment maneuvers, and high positive end-expiratory pressure for acute lung injury and acute respiratory distress syndrome: a randomized controlled trial. JAMA 2008; 299: 637-645.

28 Mercat A, Richard JC, Vielle B, et al. Positive end-expiratory pressure setting in adults with acute lung injury and acute respiratory distress syndrome: a randomized controlled trial. JAMA 2008; 299: 646-655.

29 Gainnier M, Roch A, Forel JM, et al. Effect of neuromuscular blocking agents on gas exchange in patients presenting with acute respiratory distress syndrome. Crit Care Med 2004; 32: 113-119.

30 Forel JM, Roch A, Marin V, et al. Neuromuscular blocking agents decrease inflammatory response in patients presenting with acute respiratory distress syndrome. Crit Care Med 2006; 34: 2749-2757.

31 Papazian L, Forel JM, Gacouin A, et al. Neuromuscular blockers in early acute respiratory distress syndrome. N Engl J Med 2010; 363: 1107-1116.

32 Fan E, Dowdy DW, Colantuoni E, et al. Physical complications in acute lung injury survivors: a two-year longitudinal prospective study. Crit Care Med 2014; 42: 849-859.

33 Herridge MS, Tansey CM, Matté A, et al. Functional disability 5 years after acute respiratory distress syndrome. N Engl J Med 2011; 364: 1293-1304.

34 Slutsky AS. Neuromuscular blocking agents in ARDS. N Engl J Med 2010; 363: 1176-1180.

35 Jaber S, Petrof BJ, Jung B, et al. Rapidly progressive diaphragmatic weakness and injury during mechanical ventilation in humans. Am J Respir Crit Care Med 2011; 183: 364-371. 
36 Levine S, Nguyen T, Taylor N, et al. Rapid disuse atrophy of diaphragm fibers in mechanically ventilated humans. N Engl J Med 2008; 358: 1327-1335.

37 Jaber S, Sebbane M, Verzilli D, et al. Adaptive support and pressure support ventilation behavior in response to increased ventilatory demand. Anesthesiology 2009; 110: 620-627.

38 Sassoon CS, Zhu E, Caiozzo VJ. Assist-control mechanical ventilation attenuates ventilator-induced diaphragmatic dysfunction. Am J Respir Crit Care Med 2004; 170: 626-632.

39 Neumann P, Wrigge H, Zinserling J, et al. Spontaneous breathing affects the spatial ventilation and perfusion distribution during mechanical ventilatory support. Crit Care Med 2005; 33: 1090-1095.

40 Putensen C, Mutz NJ, Putensen-Himmer G, et al. Spontaneous breathing during ventilatory support improves ventilation-perfusion distributions in patients with acute respiratory distress syndrome. Am J Respir Crit Care Med 1999; 159: 1241-1248.

41 Yoshida T, Torsani V, Gomes S, et al. Spontaneous effort causes occult pendelluft during mechanical ventilation. Am J Respir Crit Care Med 2013; 188: 1420-1427.

42 Yoshida $\mathrm{T}$, Uchiyama A, Matsuura $\mathrm{N}$, et al. Spontaneous breathing during lung-protective ventilation in an experimental acute lung injury model: high transpulmonary pressure associated with strong spontaneous breathing effort may worsen lung injury. Crit Care Med 2012; 40: 1578-1585.

43 Richard JC, Lyazidi A, Akoumianaki E, et al. Potentially harmful effects of inspiratory synchronization during pressure preset ventilation. Intensive Care Med 2013; 39: 2003-2010.

44 Ashbaugh DG, Bigelow DB, Petty TL, et al. Acute respiratory distress in adults. Lancet 1967: 319-323.

45 Brower RG, Lanken PN, MacIntyre N, et al. Higher versus lower positive end-expiratory pressures in patients with the acute respiratory distress syndrome. N Engl J Med 2004; 351: 327-336.

46 Suter PM, Fairley HB, Isenberg MD. Optimum end-expiratory airway pressure in patients with acute pulmonary failure. N Engl J Med 1975; 292: 284-289.

47 Brochard L. Measurement of esophageal pressure at bedside: pros and cons. Curr Opin Crit Care 2014; 20: 39-46.

48 Dantzker DR, Lynch JP, Weg JG. Depression of cardiac output is a mechanism of shunt reduction in the therapy of acute respiratory failure. Chest 1980; 77: 636-642.

49 Gattinoni L, Caironi $\mathrm{P}$, Cressoni $\mathrm{M}$, et al. Lung recruitment in patients with the acute respiratory distress syndrome. N Engl J Med 2006; 354: 1775-1786.

50 Terragni PP, Filippini C, Slutsky AS, et al. Accuracy of plateau pressure and stress index to identify injurious ventilation in patients with acute respiratory distress syndrome. Anesthesiology 2013; 119: 880-889.

51 Caironi $\mathrm{P}$, Cressoni $\mathrm{M}$, Chiumello $\mathrm{D}$, et al. Lung opening and closing during ventilation of acute respiratory distress syndrome. Am J Respir Crit Care Med 2010; 181: 578-586.

52 Crotti S, Mascheroni D, Caironi P, et al. Recruitment and derecruitment during acute respiratory failure: a clinical study. Am J Respir Crit Care Med 2001; 164: 131-140.

53 Jonson B, Richard JC, Straus C, et al. Pressure-volume curves and compliance in acute lung injury: evidence of recruitment above the lower inflection point. Am J Respir Crit Care Med 1999; 159: 1172-1178.

54 Richard J, Brochard L, Vandelet P, et al. Respective effects of end-expiratory and end-inspiratory pressures on alveolar recruitment in acute lung injury. Crit Care Med 2003; 31: 89-92.

55 Maggiore SM, Jonson B, Richard JC, et al. Alveolar derecruitment at decremental positive end-expiratory pressure levels in acute lung injury: comparison with the lower inflection point, oxygenation, and compliance. Am J Respir Crit Care Med 2001; 164: 795-801.

56 Ranieri MV, Giuliani R, Fiore T, et al. Volume-pressure curve of the respiratory system predicts effects of PEEP in ARDS: "occlusion" versus "constant flow" technique. Am J Respir Crit Care Med 1994; 149: 19-27.

57 Eissa NT, Ranieri VM, Corbeil C, et al. Analysis of the behaviour of the respiratory system in ARDS patients: effects of flow, volume and time. J Appl Physiol 1991; 70: 2719-2729.

58 Talmor D, Sarge T, Malhotra A, et al. Mechanical ventilation guided by esophageal pressure in acute lung injury. N Engl J Med 2008; 359: 2095-2104.

59 Staffieri F, Stripoli T, De Monte V, et al. Physiological effects of an open lung ventilatory strategy titrated on elastance-derived end-inspiratory transpulmonary pressure: study in a pig model. Crit Care Med 2012; 40: 2124-2131.

60 Grasso S, Terragni P, Birocco A, et al. ECMO criteria for influenza A (H1N1)-associated ARDS: role of transpulmonary pressure. Intensive Care Med 2012; 38: 395-403.

61 Dellamonica J, Lerolle N, Sargentini C, et al. Accuracy and precision of end-expiratory lung-volume measurements by automated nitrogen washout/washin technique in patients with acute respiratory distress syndrome. Crit Care 2011; 15: R294.

62 Chiumello D, Carlesso E, Cadringher P, et al. Lung stress and strain during mechanical ventilation for acute respiratory distress syndrome. Am J Respir Crit Care Med 2008; 178: 346-355.

63 Bouhemad B, Brisson $\mathrm{H}$, Le-Guen $\mathrm{M}$, et al. Bedside ultrasound assessment of positive end-expiratory pressure-induced lung recruitment. Am J Respir Crit Care Med 2011; 183: 341-347.

64 Mekontso Dessap A, Boissier F, Leon R, et al. Prevalence and prognosis of shunting across patent foramen ovale during acute respiratory distress syndrome. Crit Care Med 2010; 38: 1786-1792.

65 Bein $\mathrm{T}$, Weber-Carstens S, Goldmann A, et al. Lower tidal volume strategy $(\sim 3 \mathrm{ml} / \mathrm{kg})$ combined with extracorporeal $\mathrm{CO}_{2}$ removal versus 'conventional' protective ventilation $(6 \mathrm{ml} / \mathrm{kg})$ in severe ARDS: the prospective randomized Xtravent-study. Intensive Care Med 2013; 39: 847-856.

66 Terragni PP, Del Sorbo L, Mascia L, et al. Tidal volume lower than $6 \mathrm{ml} / \mathrm{kg}$ enhances lung protection: role of extracorporeal carbon dioxide removal. Anesthesiology 2009; 111: 826-835. 